\title{
Outcomes and revision rates following multilevel anterior cervical discectomy and fusion
}

\author{
Joseph L. Laratta ${ }^{1}$, Hemant P. Reddy ${ }^{2}$, Kelly R. Bratcher ${ }^{1}$, Katlyn E. McGraw ${ }^{1}$, Leah Y. Carreon ${ }^{1}$, \\ R. Kirk Owens II $^{1}$
}

${ }^{1}$ Norton Leatherman Spine Center, Louisville, KY, USA; ${ }^{2}$ Northeast Ohio Medical University, Rootstown, OH, USA

Contributions: (I) Conception and design: LY Carreon, RK Owens 2nd; (II) Administrative support: HP Reddy, KR Bratcher, KE McGraw; (III) Provision of study materials or patients: LY Carreon, RK Owens 2nd; (IV) Collection and assembly of data: KR Bratcher, KE McGraw; (V) Data analysis and interpretation: JL Laratta, HP Reddy, LY Carreon, RK Owens 2nd; (VI) Manuscript writing: All authors; (VII) Final approval of manuscript: All authors. Correspondence to: Joseph L. Laratta, MD. Norton Leatherman Spine Center, 210 E Gray Street, Suite 900, Louisville, KY 40202, USA.

Email: larattaj@gmail.com.

Background: Anterior cervical discectomy and fusion (ACDF) for cervical degenerative disease is an
accepted treatment for symptomatic cervical radiculopathy and myelopathy. One- and two-level fusions are
much more common and more widely studied. Outcomes and revision rates for three- and four-level ACDF
have not been well described. The purpose of this study is to report on clinical outcomes and revision rates
following multilevel ACDF. Methods: Patients who underwent three- or four-level anterior cervical discectomy with plate fixation between 2006 and 2011 from a single-center multi-surgeon practice for symptomatic cervical degenerative disease were identified. Improvements in neck disability index (NDI), neck and arm pain scores two years after surgery and revision rates were analyzed.

Results: Forty-six patients with a mean age of 55.9 years were included in the analysis. Twenty-one (46\%) were male, 10 (22\%) were smokers. Forty-one (89\%) underwent three-level fusion and 5 (11\%) underwent four-level fusion. NDI improved from 34.46 at baseline to 25.47 at 2 years. Neck pain improved from 7.04 at baseline to 3.95 and arm pain improved from 6.24 to 3.09 at 2 year follow up. Sixteen patients (35\%) returned to surgery within 2 years with 11 of these patients $(24 \%)$ returning for non-union. The average number of days to revision surgery was $750.6 \pm 570.3$ days.

Conclusions: Patients undergoing three- and four-level ACDF for multilevel cervical disease demonstrate substantial improvement in outcomes. However, the two-year revision rate is relatively high at $35 \%$ with the majority of these patients returning due to non-union.

Keywords: Anterior cervical discectomy and fusion (ACDF); revision; outcome; pseudarthrosis; multilevel; radiculopathy

Submitted Jun 01, 2018. Accepted for publication Jun 15, 2018.

doi: $10.21037 /$ jss.2018.06.16

View this article at: http://dx.doi.org/10.21037/jss.2018.06.16

\section{Introduction}

Anterior cervical discectomy and fusion (ACDF) with plate fixation is the current standard treatment for symptomatic cervical myelopathy and radiculopathy unresponsive to non- operative care, especially when kyphosis prevents dorsal cord migration and adequate ventral cord decompression with posterior procedures (1). ACDF utilizes a dissection through intermuscular planes which minimizes muscle 
Table 1 Summary of demographic data for patients undergoing multilevel ACDF

\begin{tabular}{lc}
\hline Characteristic & $\mathrm{N}(\%)$ \\
\hline Age, years, mean (SD) & $55.91(10.12)$ \\
Males & $21(46 \%)$ \\
Smokers & $10(22 \%)$ \\
ASA grade & \\
2 & $16(35 \%)$ \\
3 & $27(59 \%)$ \\
4 & $3(7 \%)$ \\
BMI, mean (SD) & $30.74(5.90)$ \\
\hline
\end{tabular}

ACDF, anterior cervical discectomy and fusion; ASA, American Society of Anesthesiologists; BMI, body mass index.

trauma which is commonly seen in a posterior approach. An anterior discectomy is performed with placement of an interbody graft and commonly a stabilizing anterior plate. Interbody graft may include structural allograft, structural autograft or a combination thereof with an interbody cage of titanium or polyether ether ketone (PEEK). The goal of the surgery is to decompress the spinal cord and/or nerve roots while maintaining bony integrity, spinal stability and restoring cervical lordosis $(1,2)$.

Fraser and colleagues reported $97 \%$ fusion rates for single level ACDF (3). Even without plating or the use of a post-operative orthosis, Jagannathan et al. reported a 94\% fusion rate in a retrospective study of single-level ACDF procedures (4). However, pathology occasionally extends over multiple levels complicating surgical management. In multilevel ACDFs, primary concerns relate to the increased soft tissue dissection and retraction needed for exposure, as well as the increased number of endplate surfaces required for successful fusion (5). Numerous studies in the literature have shown a significantly greater risk of non-union $(3,6,7)$, revision and complication (8) with multi-level ACDFs. The purpose of the current study is to report on clinical outcomes and revision rates following three and four level ACDF procedures.

\section{Methods}

Patients who underwent three- or four-level anterior cervical discectomy with plate fixation between 2006 and 2011 from a single-center multi-surgeon spine specialty practice for symptomatic cervical degenerative disease were identified from the surgical and outcome database. Operative technique was standard across all surgeons, including anterior annulotomy, thorough removal of all disc material, removal of cartilage with microsurgical curettes and decortication with high-speed burr with careful attention to not violate the endplates. Local autograft from removal of osteophytes and allograft were used to supplement PEEK or titanium interbody fusion cages. The operative vertebral bodies were spanned with a titanium plate in locking fashion. Standard demographic data including age, gender, and smoking data were collected. Surgical data collected included number of surgical levels, American Society of Anesthesiologists (ASA) Grade, operative time, estimated blood loss and length of hospital stay. Patient reported outcomes (PROs) included the neck disability index (NDI), numeric rating scales (0 to 10) neck and arm pain pre-operatively and at one and two years after surgery were also collected. Any complications and subsequent need for a revision surgery were identified from review of medical records.

Statistical analyses were performed with SPSS (IBM v23.0, Chicago, IL, USA). Un-adjusted univariate analysis was performed using independent sample $t$-test for continuous data and Chi-squared or Fisher exact tests for categorical variables. Correlations between continuous variables were examined using the Pearson correlation coefficient test. Statistical significance was defined as a $\mathrm{P}<0.05$.

This study obtained ethics approval from the University of Louisville Institutional Board (study number 14.1036) and the Norton Healthcare Office of Research Administration (study number 14.N0265).

\section{Results}

\section{Demographics}

Forty-six patients with a mean age of $55.9 \pm 10.12$ years were included in the analysis. Twenty-one (46\%) were male, $10(22 \%)$ were smokers and average BMI was $30.74 \pm 5.90$. Of the 46 patients who underwent three or four level ACDF, there were 41 (89\%) three level and 5 (11\%) four level ACDF procedures. There were $16(35 \%)$ patients classified ASA Grade II, 27 (59\%) ASA Grade III, and 3 (7\%) patients ASA grade IV (Table 1).

\section{Perioperative parameters}

Mean surgical time was $176.52 \pm 43.01$ minutes. Mean 
Table 2 Summary of perioperative data for patients undergoing multilevel ACDF

\begin{tabular}{lc}
\hline Perioperative characteristics & Data \\
\hline Number of levels, N (\%) & $41(89 \%)$ \\
3 level ACDF & $5(11 \%)$ \\
4 level ACDF & \\
Perioperative factors, mean (SD) & $106.67(85.14)$ \\
Estimated blood loss, cc & $176.52(43.01)$ \\
Operative time, minutes & $2.57(1.54)$ \\
Hospital length of stay, days & \\
Discharge disposition, N (\%) & $43(94 \%)$ \\
Home & $3(6 \%)$ \\
Rehab &
\end{tabular}

ACDF, anterior cervical discectomy and fusion.

Table 3 Summary of PROs for multilevel ACDF

\begin{tabular}{ll}
\hline Characteristic & Mean (SD) \\
\hline NDI & $34.46(13.99)$ \\
Baseline & $23.63(13.30)$ \\
12 months & $25.47(19.00)$ \\
24 months & \\
Neck pain & $7.04(2.01)$ \\
Baseline & $4.69(3.19)$ \\
12 months & $3.95(3.01)$ \\
24 months & \\
Arm pain & $6.24(2.39)$ \\
Baseline & $4.71(3.34)$ \\
12 months & $3.09(2.99)$ \\
24 months
\end{tabular}

PRO, patient reported outcome; ACDF, anterior cervical discectomy and fusion; NDI, neck disability index.

estimated blood loss was $106.67 \pm 85.14$ cc. Mean length of stay was to be $2.57 \pm 1.54$ days. Forty-three $(94 \%)$ patients were discharged home and $3(6 \%)$ patients were discharged to a rehab facility (Table 2).

\section{Patient-reported outcomes}

At baseline, the mean NDI score was $34.46 \pm 13.99$. At
Table 4 Summary of complications and re-operation for multilevel $\mathrm{ACDF}$

\begin{tabular}{lc}
\hline Summary of complication and re-operations & $\mathrm{N}(\%)$ \\
\hline Complication & $1(2 \%)$ \\
Bradycardia & $1(2 \%)$ \\
Postoperative throat swelling & $1(2 \%)$ \\
Respiratory distress & $16(35 \%)$ \\
Return to surgery & $11(24 \%)$ \\
Nonunion & $3(7 \%)$ \\
Removal of instrumentation & $2(4 \%)$ \\
ASD & $1(2 \%)$ \\
Nonunion and ASD & $750.60(570.27)$ \\
Days between index and revision, mean (SD) & \\
\hline ACDF, anterior cervical discectomy and fusion; ASD, adjacent \\
segment degeneration.
\end{tabular}

12 months follow-up, NDI score improved to $23.63 \pm 13.30$ and was $25.47 \pm 19.00$ at 24 months (Table 1). At baseline, neck pain was $7.04 \pm 2.01$. At 12 months follow-up, neck pain score improved to $4.69 \pm 3.19$ and to $3.95 \pm 3.01$ at 24 months follow-up. At baseline, arm pain score was $6.24 \pm 2.39$. At 12 months follow-up, arm pain score improved to $4.71 \pm 3.34$ and to $3.09 \pm 2.99$ at 24 months follow-up (Table 3).

\section{Complications and reoperations}

Complications occurred in $3(6.5 \%)$ patients. One patient had symptomatic bradycardia, 1 patient had post-operative throat swelling and 1 patient had respiratory distress. Sixteen patients $(35 \%)$ returned to surgery within 2 years with 11 of these patients (24\%) returning for non-union. Three (7\%) patients returned for hardware removal, 2 (4\%) patients returned for adjacent segment disease (ASD), and $1(2 \%)$ patient returned for non-union plus ASD. The mean number of days to revision surgery was $750.60 \pm 570.27$ days from the index procedure (Table 4).

\section{Discussion}

ACDF with plate fixation is the current standard surgical treatment for cervical spondylosis with radiculopathy. Unfortunately, there is a shortage of high quality evidence examining multilevel ACDF. The current study reports on the outcomes and revision rates of multilevel ACDF for 
the treatment of cervical radiculopathy. Understanding the efficacy of multilevel ACDF procedures will permit more educated and evidence-based surgical decision making in the treatment of cervical disorders, as well as appropriate patient counseling.

Although the rate of pseudarthrosis reported in the literature following multilevel ACDF is widely variable, the pseudarthrosis rate of $24 \%$ in the current study is within the range described in the literature. In a retrospective review of 46 patients, Papadopoulos and colleagues reported a pseudarthrosis rate of 4\% (9). Li et al. compared ACDF and cervical corpectomy for four level cervical spondylotic myelopathy (CSM) and demonstrated a $9.7 \%$ pseudarthrosis rate in the ACDF cohort (2). In a similar comparison study of ACDF and cervical corpectomy, Song and colleagues reported a $12 \%$ pseudarthrosis rate (10).

The difference in pseudarthrosis rates between two, three, and four level procedures has been described in the literature. A systematic review by Jiang et al. reported pseudarthrosis rates of $37.3 \%$ for three-level and 33.3\% for four-level procedures without significant difference between the two (7). In contrast, the pseudarthrosis rate of $18.4 \%$ for two-level procedures was significantly different than both three- and four-level procedures (7). A study of 89 patients undergoing ACDF with minimum 2 year followup reported significantly higher rates of pseudarthrosis in two level (OR 1.844) and three level (OR 3.147) procedures when compared to single level procedures (6).

Overwhelmingly greater reoperation rates have been reported with multilevel ACDF when compared to single or two-level procedures. The reported increase in pseudarthrosis seen in multilevel ACDF is due to greater graft-bone interfaces required for fusion and altered biomechanics at several segments $(7,11,12)$. In the current study, we report a $35 \%$ reoperation rate: $26 \%$ for pseudarthrosis and $6 \%$ for ASD. However, the rates of ASD, pseudarthrosis, and reoperation are quite varied across the literature. Veeravagu and colleagues performed an analysis of an administrative database and reported $10.7 \%$ revision surgery rate for multilevel ACDF-significantly greater than the $9.13 \%$ for single level ACDF (8). Moreover, multilevel ACDF patients had greater all cause hospital readmission (OR 1.2, $\mathrm{P}=0.007)$ and more complications (OR 1.3, $\mathrm{P}=0.0003$ ) (8). A systematic review reported $27.33 \%$ for adjacent segment degeneration in one or two level ACDF and $29.73 \%$ in three, four, or five level procedures (13). In three level ACDF procedures, Papadopoulos et al. reported adjacent level disease in $25 \%$ of patients and with $4.5 \%$ of patients requiring additional surgery (9). Additionally, Song et al. reported 64\% adjacent level disease with $8 \%$ revision surgery in a comparison study of ACDF versus anterior cervical corpectomy and fusion for multilevel CSM (10).

In the current study, we report an overall complication rate of $6.5 \%$, with the most common complications including bradycardia, post-operative throat swelling and respiratory distress. Fountas and colleagues performed a retrospective study of 1,104 patients receiving ACDF surgery. The authors reported a $19.3 \%$ complication rate: $9.5 \%$ due to postoperative dysphagia, $5.6 \%$ due to hematoma and $3.1 \%$ due to recurrent laryngeal nerve palsy (5). Furthermore, they found that 3-level ACDF procedures resulted in greater rates of postoperative dysphagia compared to 1- or 2-level procedures (5). Similarly, Veeravagu et al. reported significantly greater complications in multilevel versus single level ACDF (OR 1.4) (8).

Despite the increased risk of complications, the current study suggests that multilevel procedures are effective. The NDI scores in our series improved from 34.46 at baseline to 25.47 at 24 months after surgery, an 8.99 overall mean improvement. Li and colleagues compared multilevel ACDF and anterior cervical corpectomy and fusion in patients with four-level CSM. The authors reported a significant decrease in NDI scores from $26.5 \pm 3.0$ to $13.1 \pm 3.2$ in the ACDF cohort (2). In a similar comparison study of ACDF and anterior cervical corpectomy and fusion, Lin et al. reported an NDI improvement from $12.56 \pm 3.0$ preoperatively to $3.44 \pm 1.7$ postoperatively (14). The authors concluded that NDI improvement was better in ACDF, but clinical outcomes were not significantly different between the two cohorts (14).

The limitations of this study are largely related to the small sample size of 46 patients. Unfortunately, the overwhelming majority of ACDF procedures were three levels. The limited number of four level procedures limits the ability to further stratify data to compare three and four level ACDF procedures with statistical significance. Lastly, because all patients within this study were operated on at a single institution by a single surgeon, the generalizability may be limited.

ACDF is an effective surgical option for treating CSM across multiple levels. However, multilevel ACDF is associated with greater reoperation, complication and pseudarthrosis rates when compared to single level ACDF. Spine surgeons must recognize these factors and educate patients appropriately when indicating the appropriate procedure for cervical radiculopathy. In the current study, patients undergoing three- and four-level ACDF 
for multilevel cervical disease demonstrate substantial improvement in outcomes; however, the $35 \%$ two-year revision rate is exceedingly high.

\section{Acknowledgements}

None.

\section{Footnote}

Conflicts of Interest: Dr. JL Laratta reports consulting for Evolution Spine and research support from Orthopaedic Science Research Foundation and Norton Healthcare. Dr. LY Carreon reports consulting for AO Spine, trips/travel for Center for Spine Surgery and Research of the University of Southern Denmark and University of Louisville Institutional Review Board, and research support from the Scoliosis Research Society, Orthopedic Educational Research Fund, Integra and Pfizer. Dr. R. Kirk Owens reports consulting for Medtronic, Nuvasive and Alphatec, trips/travel from Depuy Synthes, Medtronic, and Nuvasive, research support from Pfizer and Integra, and fellowship support from Norton Healthcare. The other authors have no conflicts of interest to declare.

Ethical Statement: This study obtained ethics approval from the University of Louisville Institutional Board (Study number 14.1036) and the Norton Healthcare Office of Research Administration (Study number 14.N0265).

\section{References}

1. Hillard VH, Apfelbaum RI. Surgical management of cervical myelopathy: indications and techniques for multilevel cervical discectomy. Spine J 2006;6:242S-51S.

2. Li Z, Huang J, Zhang Z, et al. A Comparison of Multilevel Anterior Cervical Discectomy and Corpectomy in Patients With 4-level Cervical Spondylotic Myelopathy: a Minimum 2-year Follow-up Study: Multilevel Anterior Cervical Discectomy. Clin Spine Surg 2017;30:E540-6.

3. Fraser JF, Härtl R. Anterior approaches to fusion of the

Cite this article as: Laratta JL, Reddy HP, Bratcher KR, McGraw KE, Carreon LY, Owens RK 2nd. Outcomes and revision rates following multilevel anterior cervical discectomy and fusion. J Spine Surg 2018;4(3):496-500. doi: 10.21037/ jss.2018.06.16 cervical spine: a metaanalysis of fusion rates. J Neurosurg Spine 2007;6:298-303.

4. Jagannathan J, Shaffrey CI, Oskouian RJ, et al. Radiographic and clinical outcomes following single-level anterior cervical discectomy and allograft fusion without plate placement or cervical collar. J Neurosurg Spine 2008;8:420-8.

5. Fountas KN, Kapsalaki EZ, Nikolakakos LG, et al. Anterior cervical discectomy and fusion associated complications. Spine (Phila Pa 1976) 2007;32:2310-7.

6. Lee DH, Cho JH, Hwang CJ, et al. What is the Fate of Pseudarthrosis Detected 1 Year After Anterior Cervical Discectomy and Fusion? Spine (Phila Pa 1976) 2018;43:E23-8.

7. Jiang SD, Jiang LS, Dai LY. Anterior cervical discectomy and fusion versus anterior cervical corpectomy and fusion for multilevel cervical spondylosis: a systematic review. Arch Orthop Trauma Surg 2012;132:155-61.

8. Veeravagu A, Cole T, Jiang B, et al. Revision rates and complication incidence in single- and multilevel anterior cervical discectomy and fusion procedures: an administrative database study. Spine J. 2014;14:1125-31.

9. Papadopoulos EC, Huang RC, Girardi FP, et al. Threelevel anterior cervical discectomy and fusion with plate fixation: radiographic and clinical results. Spine (Phila $\mathrm{Pa}$ 1976) 2006;31:897-902.

10. Song KJ, Lee KB, Song JH. Efficacy of multilevel anterior cervical discectomy and fusion versus corpectomy and fusion for multilevel cervical spondylotic myelopathy: a minimum 5-year follow-up study. Eur Spine J 2012;21:1551-7.

11. Wang JC, McDonough PW, Endow K, et al. The effect of cervical plating on single-level anterior cervical discectomy and fusion. J Spinal Disord 1999;12:467-71.

12. Emery SE, Bolesta MJ, Banks MA, et al. Robinson anterior cervical fusion comparison of the standard and modified techniques. Spine (Phila Pa 1976) 1994;19:660-3.

13. Carrier CS, Bono CM, Lebl DR. Evidence-based analysis of adjacent segment degeneration and disease after ACDF: a systematic review. Spine J 2013;13:1370-8.

14. Lin Q, Zhou X, Wang X, et al. A comparison of anterior cervical discectomy and corpectomy in patients with multilevel cervical spondylotic myelopathy. Eur Spine J 2012;21:474-81. 